\title{
The Effects of Normalization, Transformation, and Rarefaction on Clustering of OTU Abundance
}

David Molik,dmolik@nd.edu, Department of Biology, University of Notre Dame, Notre Dame, United States of America

Michael Pfrender, mpfrende@nd.edu, Department of Biology, University of Notre Dame, Notre Dame, United States of America

Scott Emrich, semrich@nd.edu, Department of Electrical Engineering and Computer Science, The University of Tennessee Knoxville, Knoxville, United States of America

\section{Introduction}

Metagenomic clustering presents a unique opportunity to associate and understand communities. Working with Operational Taxonomic Units (OTUs), however, often requires a strategy for handling OTUs that may be over or under represented in a given sample, which is thought of as "erroneous". PCR amplification, for example, is known to sometimes non-linearly over-represent more common species Gonzalez et al. 2012. Strategies dealing with this bias include Normalization, Rarefaction, and Log Transformation. Here, we examine how methods to handle potential outlier observations affect de novo estimation of groups using both clustering and matrix factorization methods.

While log transformations may affect parametric tests O'Hara and Kotze 2010 our primary interest is in clustering, where relative abundances among samples could affect the derived clusters Weiss et al. 2017. We examine similarity between the adjusted OTU tables and the original ones to determine if relationships are preserved after post-processing.

We consider four methods: Bray-Curtis Similarity, de novo determined clusters by K-means and PCA, determining groups using Non-Negative Matrix Factorization (NMF). Our goal is to adjust for potential over or under representation while keeping realtive sample abudnaces as similar as possible, a problem that finds analogy in Single Cell sequencing Vallejos et al. 2017. We find that Rarefaction and then Transformation have higher mantel statistics, as a matrix correlation Legendre and Legendre 1998, in relation to an unmodified OTU table than a Normalized OTU table, and this same observation applies also to K-means. Only for NMF does Log Transformation results appear similar to an unedited table.

\section{Methods}


We estimate a Bray-Curtis distance matrix from each of four OTU tables Fig. 1 and compare these matrices with a mantel statistic Fig. 2 to see if Normalization, Rarefaction, or Log Transformation
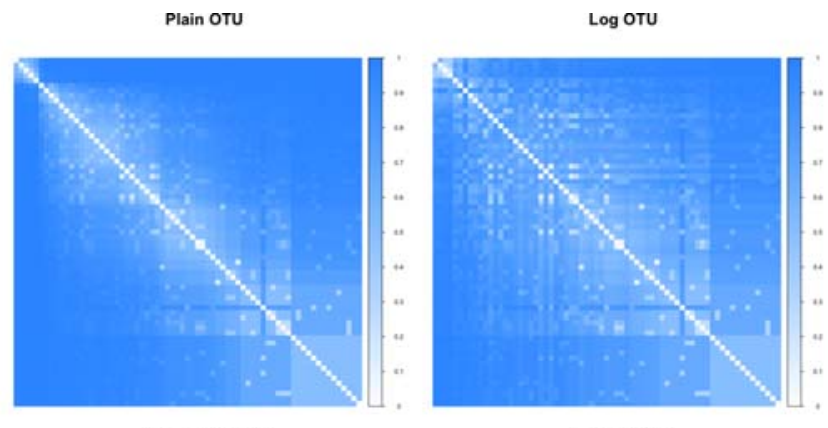

Normalized OTU

Rarefied OTU
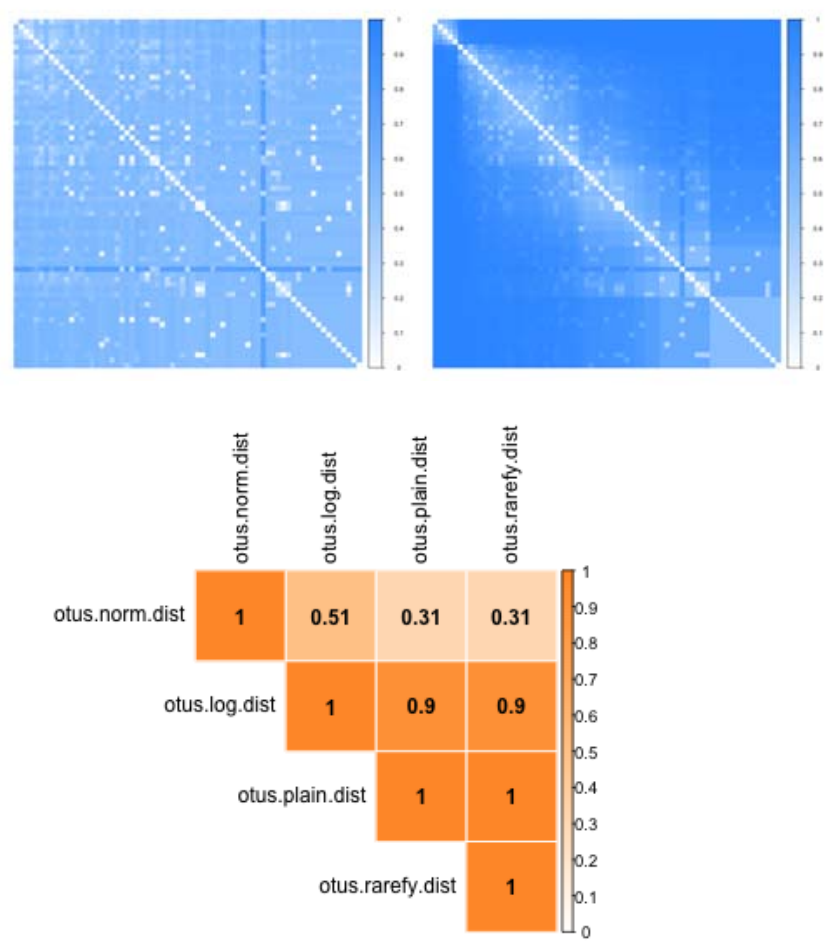

disrupts groups. Data was chosen that had three distinct sampling locations and three distinct OTU profiles Crits-Christoph et al. 2013. We used k=3 for K-means and NMF, which is described below.

\section{K-means, PCA of Abundance}


Using OTU tables we generate de novo clusters with K-means and then compare these clusters using a Jaccard-driven similarity Fig. 3. We test these clusters using a silhouette plot, which shows the distance
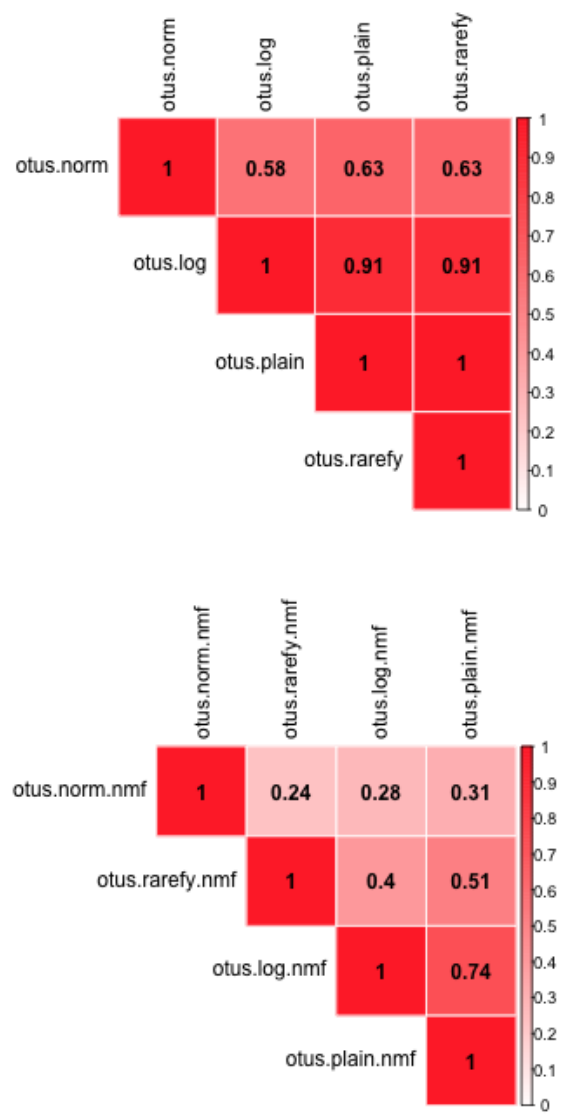

of each sample to the closest other cluster. The clusters are plotted with a PCA on abundance, using the DC algorithm Fig. 4. Rand Similarities show the same relationships between OTU tables, and when a Wilcoxon signed-rank test is conducted between all tables, log transform is seen to have the highest statistic to the un-edited OTU table Molik 2017. 

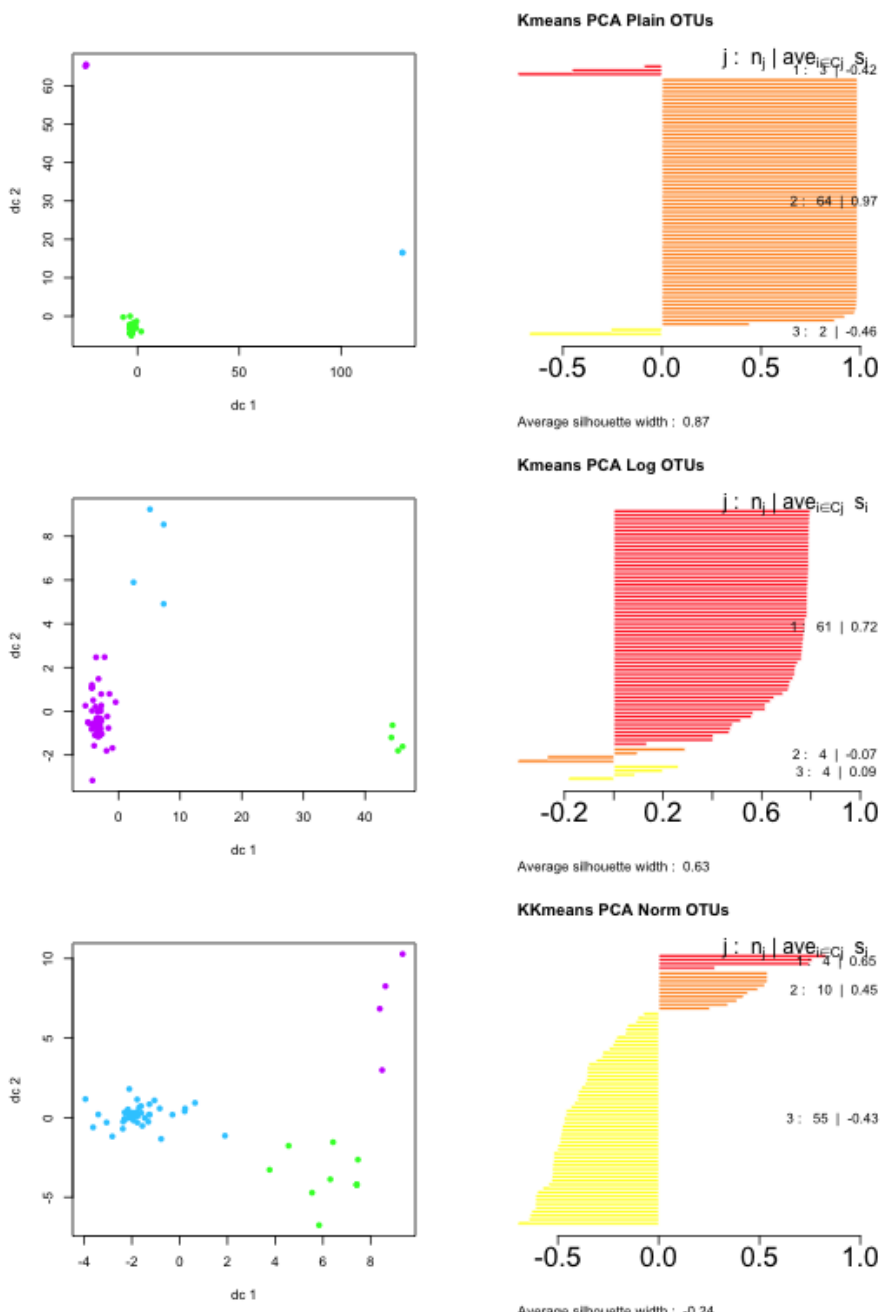

Kmeans PCA Rarefy OTUs
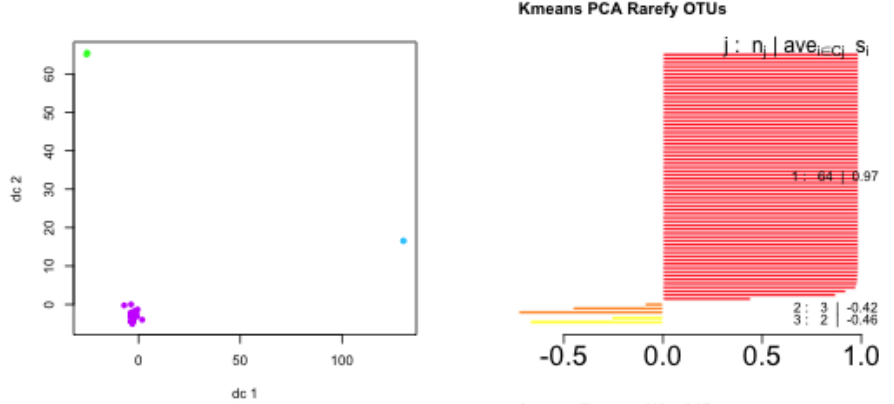

Non-negative Matrix Factorization

Using the OTU tables we generate de novo clusters in NMF and compare these clusters with Jaccard Similarity Fig. 3. NMF groups had higher silhouette coefficients than their K-means counterparts Fig. 5. All groups had an above .8 silhouette coefficient. 
bioRxiv preprint doi: https://doi.org/10.1101/259325; this version posted February 6, 2018. The copyright holder for this preprint (which was not certified by peer review) is the author/funder, who has granted bioRxiv a license to display the preprint in perpetuity. It is made available under aCC-BY 4.0 International license.
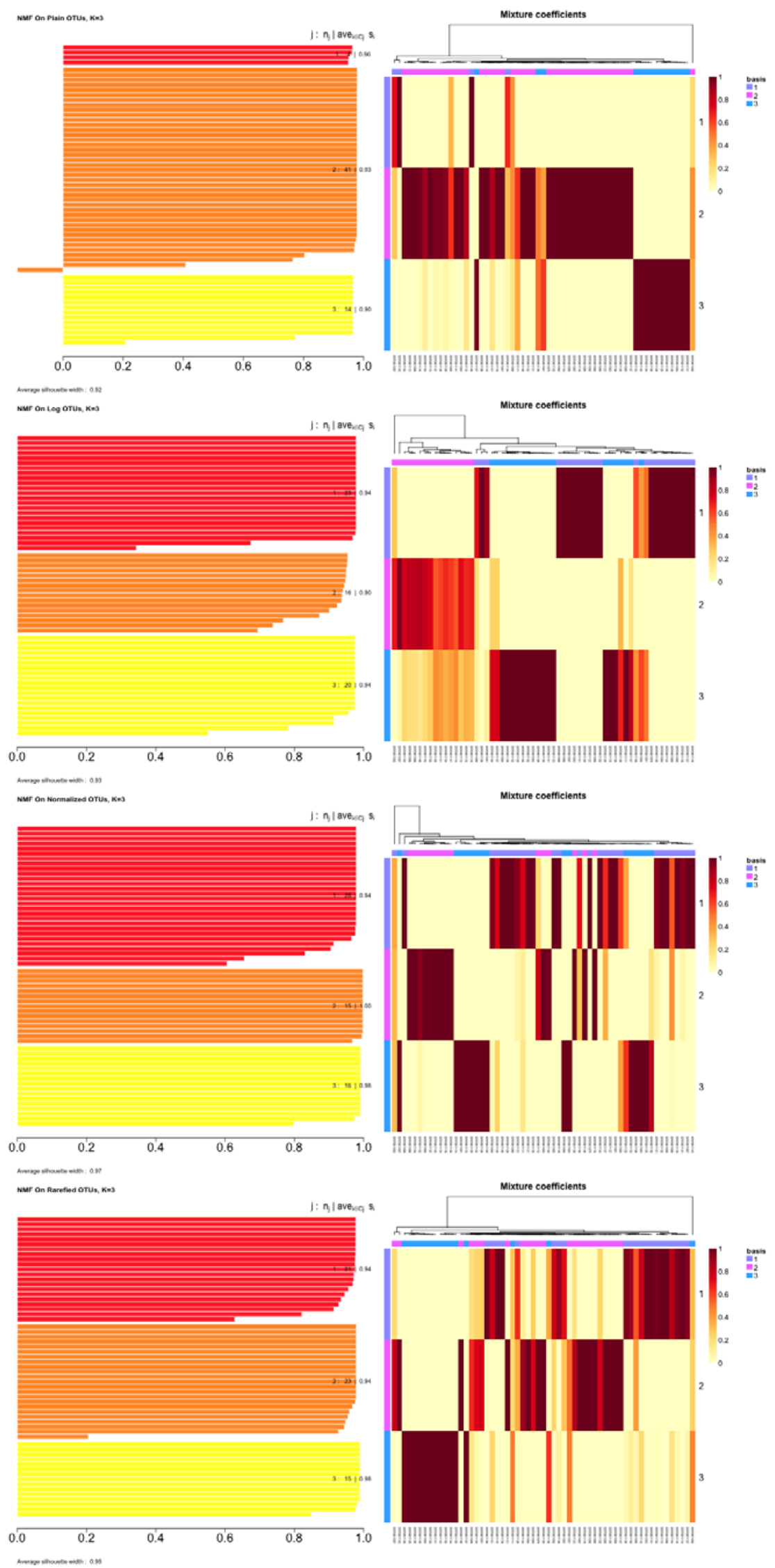

Discussion 
Distance matrices are used in hierarchical clustering. In Figure 2 we can observe that "normalized" is a fairly distinct distance matrix, and has been shown to cause a substantial difference in OTU composition Weiss et al. 2017. These differences appear using mantel statistic assessment, as well as in K-means analysis and in NMF clustering. Similarly, the expectation in Rarefaction is that relative abundances will change according to the amount of rarefaction; a presence absence OTU table will have different sample to sample relationships than a slightly scaled down OTU table. We look at the later and, as expected, our rarefied table is shown to be more similar based on a mantel test on distance matrices, as well as in PCA/K-means assessments.

The optimal method for handling outliers and erroneous abundances depends on data and application Weiss et al. 2017. In clustering and dissimilarity both rarification and log transformation seems to produce results that approximate the original OTU table; however, formatrix factorization only log transformation maintains original OTU table structure.

\section{Data and Code Availability}

Source sequence data came from National Center for Biotechnology Information Sequence Read Archive SRA accession number SRA091062 Crits-Christoph et al. 2013. Analysis was conducted in R and is available at https://github.com/status-five/The-Effects-of-Normalization-Transformation-andRarefication-on-Clustering-on-OTU-Abundance, the DOI:10.5281/zenodo.896927 version was used Molik 2017. We used the R vegan package Dixon 2003 for log transformation, normalization, and rarefaction of the OTU table. The NMF pacage was used for applying NMF Gaujoux and Seoighe 2010. The Corrplot R pacakge was used for matrices visualization V. Simko 2016. The fpc R package was used for PCA analysis and visualization Hennig 2015. The clusteval package was used for determining Jaccard similarities between clusters Ramey 2012.

\section{References}

Crits-Christoph A, Robinson CK, Barnum T, Fricke W, Davila AF, Jedynak B, McKay CP, DiRuggiero J (2013) Colonization patterns of soil microbial communities in the Atacama Desert. Microbiome 1 (1): 28. https://doi.org/10.1186/2049-2618-1-28

Dixon P (2003) VEGAN, a package of R functions for community ecology. Journal of Vegetation Science 14 (6): 927. https://doi.org/10.1658 /11009233(2003)014[0927:vaporf]2.0.co;2

Gaujoux R, Seoighe C (2010) A flexible R package for nonnegative matrix factorization . BMC Bioinformatics 11 (1): 367. https://doi.org/10.1186/1471-2105-11-367

Gonzalez J, Portillo M, Belda-Ferre P, Mira A (2012) Amplification by PCR Artificially Reduces the Proportion of the Rare Biosphere in Microbial Communities. PLoS ONE 7 (1): e29973. https://doi.org/10.1371/journal.pone.0029973

Hennig C (2015) fpc: Flexible Procedures for Clustering. 2.1-10. URL: https:/CRAN.R- 
project.org/package $=\mathrm{fpc}$

Legendre P, Legendre L (1998) Numerical ecology. Elsiver

Molik D (2017) Status-Five/The-Effects-Of-Normalization-Transformation-And- RareficationOn-Clustering-On-Otu-Abundance: Intial Release. Zenodo https://doi.org /10.5281/ZENODO.896926

O'Hara R, Kotze DJ (2010) Do not log-transform count data. Methods in Ecology and Evolution 1 (2): 118ロ122. https://doi.org/10.1111/j.2041-210x.2010.00021.x

Ramey J (2012) clusteval: Evaluation of Clustering Algorithms. 0.1. URL:

https://CRAN.R-project.org/package=clusteval

Vallejos CA, Risso D, Scialdone A, Dudoit S, Marioni JC (2017) Normalizing single-cell

RNA sequencing data: challenges and opportunities. Nature Methods 14 (6): $565 \square 571$. https://doi.org/10.1038/nmeth.4292

V. Simko TW (2016) R package 'corrplot': visualization of a correlation matrix,. Matrix R Packag (77).

Weiss S, Xu ZZ, Peddada S, Amir A, Bittinger K, Gonzalez A, Lozupone C, Zaneveld J, Vázquez-Baeza Y, Birmingham A, Hyde E, Knight R (2017) Normalization and microbial differential abundance strategies depend upon data characteristics. Microbiome 5 (1): $1 \square 18$. https://doi.org/10.1186/s40168-017-0237-y 